\title{
Neuromyositis: A Rare Extramuscular Manifestation of Dermatomyositis
}

\author{
Chan Keol Park, Su-Jin Yoo, In Seol Yoo, Jinhyun Kim, Seung Cheol Shim, Seong Wook Kang \\ Division of Rheumatology, Department of Internal Medicine, Chungnam National University Hospital, Daejeon, Korea
}

\begin{abstract}
Dermatomyositis (DM) and polymyositis (PM) are representative idiopathic inflammatory myopathies characterized by symmetric and progressive proximal muscle weakness. Especially, DM is identified by characteristic skin lesions and has many extramuscular manifestations including various cardiac abnormalities, interstitial lung disease, and malignancy. However, involvement of peripheral nervous system in DM/PM is very rare and less known. The term "Neuromyositis" was introduced by Senator in 1893 to describe the concomitant involvement of the peripheral nervous system in DM/PM. Since then, a very few cases of neuromyositis have been reported mainly in the United States and Europe. Therefore, the pathogenetic mechanism and disease progression are unclear. In recent years, a few more cases were reported in Asia, specifically, China and Japan; however, none in Korea. Here, we describe a case of DM-associated neuromyositis in a 42-year-old man in Korea and review previous publications through literature research. (J Rheum Dis 2019;26:211-218)
\end{abstract}

Key Words. Dermatomyositis, Peripheral nervous system diseases, Nerve conduction, Electromyography

\section{INTRODUCTION}

Dermatomyositis (DM) and polymyositis (PM) are two major groups of idiopathic inflammatory myopathies (IIM) that are characterized by chronic inflammation in the skeletal muscle, resulting in muscle weakness $[1,2]$. Especially, DM is identified by characteristic cutaneous manifestations such as heliotrope rash, Gottron's papules, cuticular changes, a photodistributed erythema or poikiloderma, a scaly alopecia and hyperkeratosis of the palms and fingers, known as "mechanic's hands" [3]. IIM have many extramuscular manifestations which include dysphagia, various cardiac abnormalities, interstitial lung disease (ILD) and malignancy. Especially, the frequency of cancer development is definitely increased in DM and slightly increased in PM [2]. However, peripheral neuropathy is very rarely reported during the course of DM/PM and less known. Concomitant involvement of a peripheral nervous system in DM/PM has been known as neuro- myositis which was first introduced by Senator in 1893 [4]. A few cases of a peripheral neuropathy in DM/PM have been reported; however, even a single study was not conducted yet to examine peripheral nerve in a large population diagnosed with DM/PM. Consequently, the clinical presentation and pathogenetic mechanism of neuromyositis are unclear. In addition, well-defined diagnostic criteria of this entity are still controversial. After the classical etiologies of peripheral neuropathies are ruled out, the diagnosis of neuromyositis can be accepted. Previously, Onder et al. [5] reported the neuropathic involvement in 8 patients diagnosed with DM/PM. Although the electrophysiologic studies showed all $8 \mathrm{pa}-$ tients with neuropathic involvement, only 1 patient diagnosed with neuromyositis because the others had comorbidities that could cause neuropathies, such as malignancies (e.g., breast cancer and lung cancer), other rheumatic diseases (e.g., Sjögren's syndrome, scleroderma, and familial Mediterranean fever), and diabetes mellitus.

Received : December 26, 2018, Revised : February 18, 2019, Accepted : March 7, 2019

Corresponding to : Seong Wook Kang (D)http://orcid.org/0000-0002-0076-0822

Division of Rheumatology, Department of Internal Medicine, Chungnam National University Hospital, 282 Munhwa-ro, Jung-gu, Daejeon 35015, Korea. E-mail : kangsw@cnuh.co.kr 
Here, we report a case of DM-associated neuromyositis in a 42-year-old man and review the previous publications through literature research.

\section{CASE REPORT}

A 42-year-old man visited our clinic with limb weakness and skin lesions on the face and hands. His symptoms occurred 3 weeks before visiting our clinic. His upper and lower extremities revealed grade 4- proximal muscle weakness symmetrically on manual muscle test (MMT) (MMT grade 4- indicates full range of motion against gravity, with less than moderate but more than minimum resistance) (Table 1). He also exhibited DM-associated skin lesions such as erythematous rashes on the nose and forehead, Gottron's papules on the extensor surfaces over the metacarpophalangeal joints and proximal interphalangeal joints of both hands, and roughening and cracking on the skin of the tips and sides of the fingers (mechanic's hands).

To identify the cause of his symptoms, we performed various tests. Laboratory tests showed a white blood cell count of $4,480 / \mu \mathrm{L}$, hemoglobin level of $14.7 \mathrm{~g} / \mathrm{dL}$, platelet count of $171,000 / \mu \mathrm{L}$, erythrocyte sedimentation rate of $34 \mathrm{~mm} / \mathrm{hr}$, and C-reactive protein level of $0.1 \mathrm{mg} / \mathrm{dL}$. The levels of aspartate aminotransferase, alanine aminotransferase, and lactate dehydrogenase were 50, 28, and $598 \mathrm{U} / \mathrm{L}$, respectively $(0 \sim 37 / 0 \sim 41 / 200 \sim 400 \mathrm{U} / \mathrm{L})$. Creatine kinase (CK) levels were elevated to $344 \mathrm{U} / \mathrm{L}$ (56 $244 \mathrm{U} / \mathrm{L})$. Viral serologic test results for hepatitis B, hepatitis $\mathrm{C}$, and human immunodeficiency virus were negative. The patient also tested negative for tumor markers, such as carbohydrate antigen 19-9, carcinoembryonic antigen, and alpha-fetoprotein. Thyroid function tests, hemoglobin A1c levels, electrolytes, blood urea nitrogen levels, and serum creatinine levels were also normal. Antinuclear antibody $(\mathrm{Ab})$ tests indicated weak positivity with a speckled pattern. Rheumatoid factor, anti-CCP Ab, anti-RNP Ab, and anti-Jo1 Ab were not detected, but anti-Ro52 Ab and anti-MDA5 Ab were positive among myositis specific antibodies.

Unusually, although there was definite muscle weakness on physical examination, electromyography (EMG) of the proximal muscles of the upper and lower ex-

Table 1. Summary of clinical and laboratory findings of the present case

\begin{tabular}{|c|c|c|c|c|}
\hline Variable & $\begin{array}{c}\text { At the time of } \\
\text { dermatomyositis diagnosis }\end{array}$ & $\begin{array}{l}\text { At the time of } \\
\text { neuromyositis diagnosis }\end{array}$ & $\begin{array}{l}\text { After recovery of } \\
\text { neuromyositis }\end{array}$ & $\begin{array}{l}\text { Reference } \\
\text { value }\end{array}$ \\
\hline \multicolumn{5}{|l|}{ Clinical findings } \\
\hline \multicolumn{5}{|l|}{ MMT grade (right/left) } \\
\hline Shoulder abduction & $4-/ 4-$ & $5 / 5$ & $5 / 5$ & \\
\hline Shoulder vertical flexion & $4-/ 4-$ & $5 / 5$ & $5 / 5$ & \\
\hline Hip flexion & $4-/ 4-$ & $5 / 5$ & $5 / 5$ & \\
\hline Ankle dorsiflexion & $5 / 5$ & $5 / 5$ & $3-/ 5$ & \\
\hline Ankle plantarflexion & $5 / 5$ & $5 / 5$ & $4-/ 5$ & \\
\hline Dyspnea (mMRC grade) & $0 *$ & 0 & 0 & \\
\hline \multicolumn{5}{|l|}{ Laboratory findings } \\
\hline $\mathrm{ESR}(\mathrm{mm} / \mathrm{hr})$ & 34 & 15 & 2 & $0 \sim 10$ \\
\hline $\mathrm{CRP}(\mathrm{mg} / \mathrm{dL})$ & 0.1 & 0.1 & 0.1 & $0 \sim 0.5$ \\
\hline CK $(U / L)$ & 344 & 136 & 129 & $56 \sim 244$ \\
\hline AST (U/L) & 50 & 22 & 23 & $0 \sim 37$ \\
\hline ALT (U/L) & 28 & 21 & 18 & $0 \sim 41$ \\
\hline $\mathrm{LDH}(\mathrm{U} / \mathrm{L})$ & 598 & 360 & - & $200 \sim 400$ \\
\hline \multicolumn{5}{|l|}{ Pulmonary function test } \\
\hline FVC, L (\% predicted) & $4.76(98)$ & $4.67(96)$ & $5.03(104)$ & \\
\hline FEV1, L (\% predicted) & $3.55(95)$ & $3.56(96)$ & $3.59(97)$ & \\
\hline FEV1/FVC, \% & 75 & 76 & 71 & \\
\hline DLco, $\mathrm{mL} / \mathrm{mmHg} / \mathrm{min}$ (\% predicted) & $32.5(129)$ & $37.1(150)$ & $30.8(122)$ & \\
\hline
\end{tabular}

MMT: manual muscle test, mMRC: modified Medical Research Council dyspnea scale, ESR: erythrocyte sedimentation rate, CRP: C-reactive protein, CK: creatine kinase, AST: aspartate aminotransferase, ALT: alanine aminotransferase, LDH: lactate dehydrogenase, FVC: forced vital capacity, FEV1: forced expiratory volume in 1 second, DLco: diffuse lung capacity for carbon monoxide. *Not troubled by breathlessness except on strenuous exercise. 
tremities showed normal findings. In contrast, a nerve conduction study (NCS) showed some abnormal findings. Peripheral motor NCS showed low-amplitude compound muscle action potential (CMAP) on the left abductor pollicis brevis and right extensor digitorum brevis (EDB). Furthermore, the peripheral sensory NCS showed a low-amplitude sensory nerve action potential (SNAP) and a slow conduction velocity in the left median nerve and absent SNAP on the right superficial peroneal nerve. These NCS findings suggested partial nerve injury on the left median nerve and right peroneal nerve (Table 2). However, an additional EMG study was not performed on the affected sites because there were no clinical symptoms or signs of peripheral neuropathy. Because the EMG study on the proximal muscles was normal, we conducted a muscle biopsy to diagnose DM after confirming my-

Table 2. Results of nerve conduction study

\begin{tabular}{|c|c|c|c|c|c|c|c|c|c|}
\hline \multirow{2}{*}{ Nerve } & \multicolumn{3}{|c|}{ 1st study } & \multicolumn{3}{|c|}{ 2nd study } & \multicolumn{3}{|c|}{ 3rd study } \\
\hline & Lat & Amp & $\mathrm{CV}$ & Lat & Amp & $\mathrm{CV}$ & Lat & Amp & $\mathrm{CV}$ \\
\hline \multicolumn{10}{|l|}{ Motor nerve } \\
\hline Left peroneal & $3.0 \mathrm{~ms}$ & $5.3 \mathrm{mV}$ & $47.7 \mathrm{~m} / \mathrm{s}$ & $3.92 \mathrm{~ms}$ & $3.2 \mathrm{mV}$ & $52.4 \mathrm{~m} / \mathrm{s}$ & $4.04 \mathrm{~ms}$ & $7.2 \mathrm{mV}$ & $53.4 \mathrm{~m} / \mathrm{s}$ \\
\hline Right peroneal & $3.42 \mathrm{~ms}$ & $2.8 \mathrm{mV}$ & $47.2 \mathrm{~m} / \mathrm{s}$ & $4.13 \mathrm{~ms}$ & $1.14 \mathrm{mV}$ & $48.8 \mathrm{~m} / \mathrm{s}$ & $4.90 \mathrm{~ms}$ & $3.3 \mathrm{mV}$ & $50.0 \mathrm{~m} / \mathrm{s}$ \\
\hline \multicolumn{10}{|l|}{ Sensory nerve } \\
\hline Left peroneal superficial & $2.4 \mathrm{~ms}$ & $5.7 \mu \mathrm{V}$ & $58.3 \mathrm{~m} / \mathrm{s}$ & $2.98 \mathrm{~ms}$ & $5.7 \mu \mathrm{V}$ & $47.0 \mathrm{~m} / \mathrm{s}$ & $2.69 \mathrm{~ms}$ & $13.2 \mu \mathrm{V}$ & $52.0 \mathrm{~m} / \mathrm{s}$ \\
\hline Right peroneal superficial & $0.0 \mathrm{~ms}$ & $0.0 \mu \mathrm{V}$ & $0.0 \mathrm{~m} / \mathrm{s}$ & $3.09 \mathrm{~ms}$ & $1.46 \mu \mathrm{V}$ & $45.3 \mathrm{~m} / \mathrm{s}$ & $2.51 \mathrm{~ms}$ & $7.3 \mu \mathrm{V}$ & $55.8 \mathrm{~m} / \mathrm{s}$ \\
\hline
\end{tabular}

The 1st study means a study performed at the time of dermatomyositis diagnosis. The 2 nd study means a study performed at the time of neuromyositis diagnosis. The 3rd study means a study performed after recovery of neuromyositis. Lat: latency, Amp: amplitude, CV: conduction velocity.
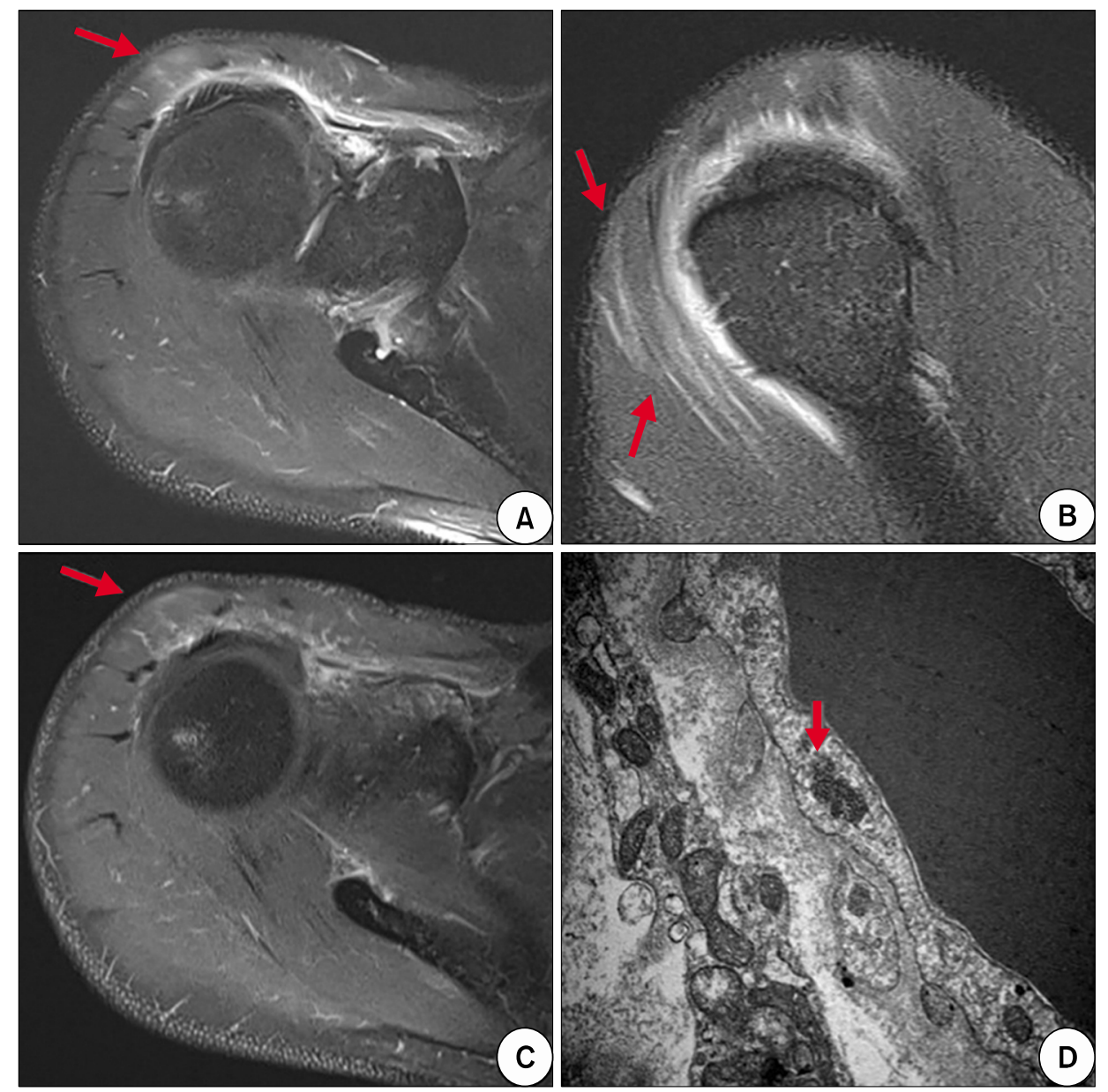

Figure 1. T2-weighted magnetic resonance images $(A, B)$ and enhanced T1-weighted magnetic resonance image (C) showed high signal intensity in right supraspinatus and anterior deltoid muscles (arrows). (D) Electron microscopy of right deltoid muscle biopsy demonstrated focal infiltration of fat globules, focal areas of myofibrillar loss, and several tubuloreticular structures (arrow) in the cytoplasm of endothelial cells, which were consistent with dermatomyositis $(\times 30,000)$. 
ositis with magnetic resonance imaging on the right upper extremity where symptoms were most severe (Figure 1). A muscle biopsy from the right deltoid muscle did not showed typical findings of DM on light microscopic examination. However, electron microscopic examination showed focal infiltration of fat globules, focal areas of myofibrillar loss, and several tubuloreticular structures in the cytoplasm of endothelial cells (Figure 1), which were consistent with DM [6].

Another important finding was that a chest computed tomography (CT) showed multifocal patchy ground glass opacity in subpleural portion of both lower lungs. These finding suggested non-specific interstitial pneumonia or cryptogenic organizing pneumonia (Figure 2). However, the patient did not complain of dyspnea and pulmonary function tests were also normal (Table 1). There were no other causes to provoke his problems such as infections, comorbidities, other rheumatic diseases, or history of drug use. Moreover, there was no evidence of malignancy in the screening tests including as tumor markers, abdominal CT, chest CT, esophagogastroduodenoscopy, or colonoscopy.

In conclusion, He was diagnosed with DM based on following findings that meet the criteria of Bohan and Peter [7]: (1) weakness in the proximal muscles of the limbs; (2) elevation of skeletal muscle enzyme levels; (3) histologic examination findings of the biopsy of the right deltoid muscle that indicates DM; and (4) skin lesions on the face and hands such as heliotrope rash, Gottron's papules, and mechanic's hands. Table 1 summarizes the important clinical and laboratory findings of the present case.

After the diagnosis of DM-ILD was established, the patient was initially treated with high-dose steroids (prednisolone at a daily dose of $1 \mathrm{mg} / \mathrm{kg}$ ). After steroid therapy, his skin rash and muscle strength improved and his elevated muscle enzyme levels returned to the normal range. However, even though there were no respiratory
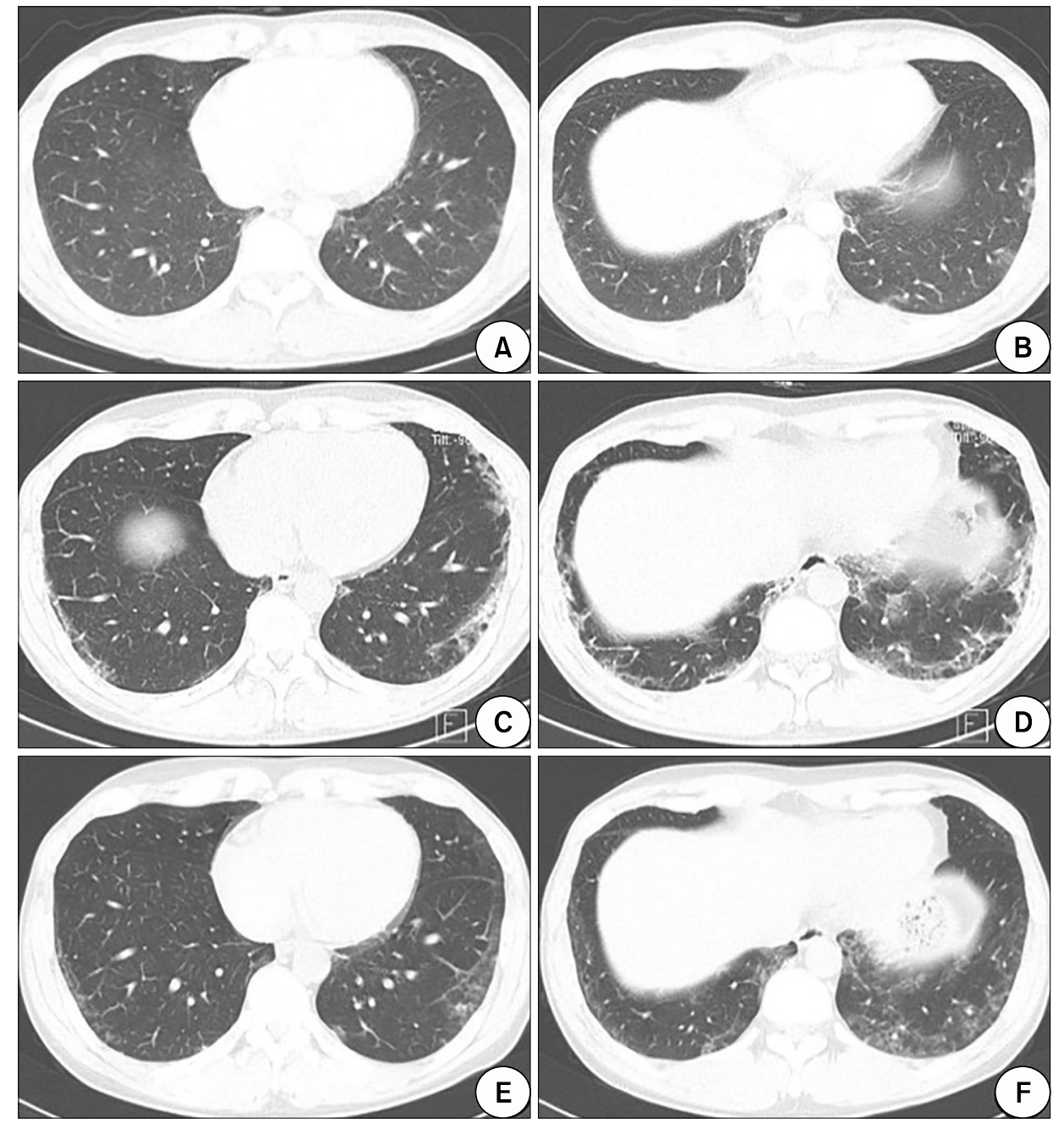

Figure 2. Initial chest computed tomography $(\mathrm{CT})$ images showed multifocal patchy ground glass opacities (GGOs) in subpleural portion of both lower lungs (A, B). Follow-up chest CT images obtained 1 month after the initiation of therapy showed aggravation to reticulation with GGOs (C, D). Follow-up chest $\mathrm{CT}$ images obtained at the time of neuromyositis diagnosis showed improving state of previous noted reticulation and GGOs (E, F). 
symptoms, such as dyspnea, follow-up chest CT performed 1 month after the initiation of steroid therapy showed worsening of ILD (Figure 2). Therefore, azathioprine $(1.5 \mathrm{mg} / \mathrm{kg} /$ day $)$ was added to the medication.

Four months after diagnosis with DM-ILD, he complained of right ankle weakness that developed gradually for 5 days. On MMT, his right ankle showed grade 3- dorsiflexion and grade 4- plantarflexion (MMT grade 3 - indicates less than full range of motion against gravity but more than 50\%) (Table 1). Other physical examinations on the right lower leg revealed normoactive deep tendon reflex and decreased sensation in the lateral side. We performed electrodiagnostic test again to determine the exact state. The peripheral motor NCS on both lower extremities showed low-amplitude CMAP on the right EDB. The peripheral sensory NCS on both lower extremities showed delayed latency and low-amplitude SNAP on the right superficial peroneal nerve (Table 2). The EMG study on the right lower extremity showed abnormal spontaneous activities in the right tibialis anterior (TA), peroneus longus, and extensor hallucis longus (EHL) and polyphasic motor unit action potential in the right EHL and TA (Table 3). These findings suggested axonal neuropathy on the right common peroneal nerve. At that time, there was no clinical evidence to suggest that DM had worsened. The strength of the proximal muscles of the upper and lower extremities still improved, and laboratory findings such as CK level were all normal (Table 1). In addition, the steroid dose was gradually reduced to $70 \%$ of the initial dose, and ILD was also improved after addition of azathioprine (Figure 2). Moreover, other causes such as trauma and infection, which may cause right ankle weakness, could not be found. Therefore, ultimately, we diagnosed the patient with neuromyositis, a peripheral nerve involvement in
DM. Because of neuromyositis, we decided to slowly taper the steroid and increase the dose of azathioprine (1.5 $\mathrm{mg} / \mathrm{kg} /$ day $\rightarrow 2.0 \mathrm{mg} / \mathrm{kg} /$ day).

Since then, right ankle weakness improved gradually and almost completely recovered after 6 months without any other further treatment. The recovery of neuromyositis was confirmed in the follow-up electrodiagnostic test. EMG and peripheral motor NCS on both lower extremities showed normal results. Only the peripheral sensory NCS on both lower extremities showed low-amplitude SNAP on the right superficial peroneal nerve (Table 2). However, this result was also significantly improved compared to that in the first test at the time of DM diagnosis as well as that in the previous test at the time of neuromyositis diagnosis. Thereafter, he has gradually reduced steroid and is now taking low-dose steroid and azathioprine. There was no recurrence of neuromyositis as well as exacerbation of DM, and other neurologic problems did not occur in any region for about 10 months after neuromyositis on his right ankle improved.

\section{DISCUSSION}

We have herein shown the extremely rare case of concomitant peripheral neuropathy and DM. In addition, we conducted literature search and compared our case with previous publications. A literature search of the PubMed database was carried out using search terms "dermatomyositis," "polymyositis," "neuromyositis," "peripheral neuropathy" and "peripheral nervous system involvement" in various combinations. Table 4 summarizes the clinical features of past cases and the present case.

Involvement of the peripheral nervous system is frequently observed in other autoimmune diseases. The prevalence rates were reported to be $40 \% \sim 50 \%$ in rheu-

Table 3. Results of electromyography study

\begin{tabular}{|c|c|c|c|c|c|c|c|}
\hline \multirow{3}{*}{ Muscle } & \multicolumn{7}{|c|}{ 2nd study } \\
\hline & \multicolumn{4}{|c|}{ Spontaneous activity } & \multicolumn{3}{|c|}{ Voluntary activity } \\
\hline & Fib & PSW & Fasc & Amp & Dur & Polyph & IP \\
\hline Right EHL & $1+$ & $1+$ & None & Normal & Normal & + & PIP-CIP \\
\hline Right PL & $2+$ & $2+$ & None & Normal & Normal & Normal & PIP-CIP \\
\hline Right TA & $2+$ & $2+$ & None & Normal & Normal & + & PIP-CIP \\
\hline
\end{tabular}

The 2nd study means a study performed at the time of neuromyositis diagnosis. EHL: extensor hallucis longus, PL: peroneus longus, TA: tibialis anterior, Fib: fibrillation potentials, PSW: positive sharp waves, Fasc: fasciculation, Amp: amplitude, Dur: duration, Polyph: polyphasic, IP: interference pattern, PIP: partial interference pattern, CIP: complete interference pattern. 


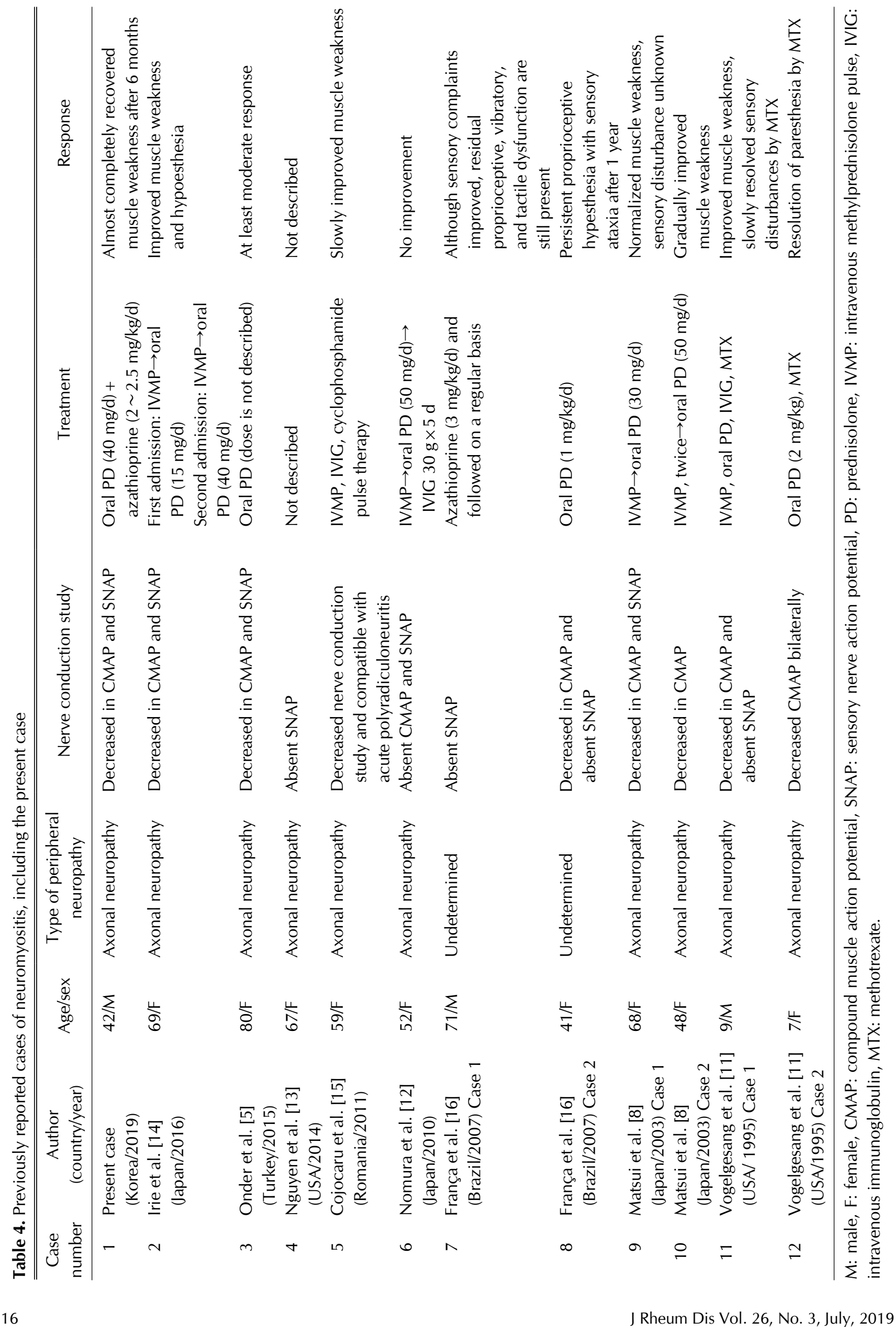


matoid arthritis, 5\% 27\% in systemic lupus erythematosus, $10 \% \sim 22 \%$ in Sjögren's syndrome, and 5\% $67 \%$ in systemic sclerosis $[8,9]$. However, the involvement of the peripheral nervous system in IIM is less known and the examination of peripheral nerves in a large population with DM/PM has not been conducted yet. Even Bohan et al. [10] who created the most commonly applied classification criteria for DM/PM, did not investigate neuropathy in 153 patients with DM/PM. Up to now, only a few neuromyositis cases have been reported.

Even though neuromyositis is defined as concomitant myopathy and neuropathy, they are generally considered to be different. However, clinical features alone may be insufficient to distinguish myopathy from neuropathy. Because of such an uncertain association, definite diagnostic criteria of this entity have not yet been established and are still controversial. In particular, the heterogeneity of associated nerve pathology and lack of unifying pathogenetic mechanism of prior case reports hinder the development of consistent diagnostic criteria for neuromyositis. Furthermore, it is difficult to diagnose neuromyositis due to the necessity of excluding other etiologic conditions causing neuropathy. In fact, Wang et al. [9] analyzed NCS in 186 patients with DM/PM in 2010 and found only 4 patients exhibited peripheral neuropathy in the absence of predisposing factors of neuropathy. Consequently, it is crucial to conduct a comprehensive evaluation in order to exclude other causes of neuropathy which can lead to the accurate diagnosis of neuromyositis. Electrodiagnostic tests, such as EMG and NCS, are useful tools in identifying neuromyositis. In addition to electrodiagnostic tests, muscle and nerve biopsies are often used to confirm the diagnosis. When the patient had a problem on his right ankle, we also performed extensive examinations to exclude the other causes and none was found. We planned to conduct nerve biopsy of the affected region if the symptoms persisted. However, it was no longer needed as the symptom showed sufficient improvement.

The pathogenetic mechanism is not clear between DM/PM and peripheral neuropathy. The histologic studies of previous publication suggest potential mechanism of neuromyositis. Matsui et al. [8] proposed a vasculitic process induced by overproduction of vascular endothelial growth factor (VEGF) in patients with DM complicated with peripheral neuropathy. Vogelgesang et al. [11] described capillary endothelial ischemia as similar to muscle pathology on nerve biopsy. On the contrary, Nomura et al. [12] demonstrated no expression of VEGF on immunohistochemical study of biopsied specimens in 2010. It is anticipated that factors other than VEGF-associated vasculitis or capillary endothelial lesions may play an important role in the development of neuromyositis. Some researchers presumed that membrane attack complex formation led to nerve involvement in patients with DM and eventually to nerve injury [13]. As such, previous histologic studies have not clearly demonstrated homogenous features; therefore, it is imperative to carry out further studies of large case series which include pathologic specimen (i.e., both muscle and nerve biopsies) in order to clarify the argument.

In our literature survey, the studies of electrodiagnostic and nerve biopsy in patients with neuromyositis exhibited that the pathologic type was predominantly axonal neuropathy, which is consistent with our patient (Table 4). These data are compatible with findings of Wang et al. [9]. In their study, distal axonal neuropathy is a predominant damage in the patients with concomitant myopathy and neuropathy. In 2016, Irie et al. [14] published a case of DM complicated with axonal neuropathy and analyzed 9 previous cases of DM with peripheral neuropathy. They also confirmed that most cases were associated with axonal neuropathy $[8,11-15]$. These results imply that neuromyositis can be anticipated when axonal neuropathy in patients with DM/PM is detected.

To date, no treatment guidelines have been proposed for neuromyositis. In previous reports, immunotherapies, such as intravenous methylprednisolone pulse (IVMP), are the most commonly used treatment for neuromyositis, based on clinical backgrounds associated with inflammatory myositis. However, the effects of immunotherapy have varied (Table 4). Some patients had a partial response to oral prednisolone and additional immunosuppressant [16], while the patient with neuromyositis in the study of Onder et al. showed a relatively good response [5]. Other studies stated that IVMP followed by oral prednisolone therapy was effective $[8,14]$. Conversely, IVMP and intravenous immunoglobulin therapy had no effect in another study [12]. In some cases, patients required a secondary immunosuppressant, such as cyclophosphamide and methotrexate in addition to IVMP $[11,15]$. In a present case, the neurologic symptom of the patient was improved just by maintaining a moderate dose of steroid and increasing a dose of azathioprine used for DM-ILD. However, because the existing treatments were not effective among some patients, it is essential to develop a well-established treatment strategy 
supported by sufficient clinical evidences.

We have summarized and analyzed previously published cases in addition to our case, except for the study of Wang et al. [9], where patients' details were not described (Table 4). Since 1893, neuromyositis has been primarily reported in the United States and Europe. In Asia, a few more cases were recently reported in China and Japan. There were no clear differences in treatments and prognosis of the neuromyositis between Western and Asian countries including our case. As mentioned, diagnostic criteria and treatment strategies for neuromyositis are not concrete at the moment. Therefore, if a large-scale study of neuromyositis including epidemiology are carried out, a more effective treatment will be available for race and geography. In that sense, our case can serve as a basis for such a research. We also emphasize that neuromyositis is one of the most important extramuscular manifestations that should be considered when treating patients with DM.

\section{SUMMARY}

Herein, we reported the case of neuromyositis associated with DM in a 42-year-old male. To the best of our knowledge, it is the first case of DM-associated neuromyositis published in Korea. Because of the lack of a well-designed study for neuromyositis, the association between IIM and peripheral neuropathy is unclear and controversial. Still, peripheral neuropathy can be one of the important extramuscular manifestations in patients with DM/PM. Therefore, further studies are much needed to elucidate the relationship between DM/PM and peripheral neuropathy.

\section{CONFLICT OF INTEREST}

No potential conflict of interest relevant to this article was reported.

\section{REFERENCES}

1. Lundberg IE, de Visser M, Werth VP. Classification of myositis. Nat Rev Rheumatol 2018;14:269-78.

2. Dalakas MC, Hohlfeld R. Polymyositis and dermatomyositis. Lancet 2003;362:971-82.

3. Callen JP. Dermatomyositis. Lancet 2000;355:53-7.

4. Senator H. Ueber acute polymyositis und neuromyositis. Deutsche Med Wochenschrift 1893;19:933-6.

5. Onder H, GökcemYıldız F, Temucin ÇM. Neuromyositis: clinical and electrophysiological study of 8 cases. Global Adv Res J Med Med Sci 2015;4:347-50.

6. De Visser M, Emslie-Smith AM, Engel AG. Early ultrastructural alterations in adult dermatomyositis. Capillary abnormalities precede other structural changes in muscle. J Neurol Sci 1989;94:181-92.

7. Bohan A, Peter JB. Polymyositis and dermatomyositis (first of two parts). N Engl J Med 1975;292:344-7.

8. Matsui N, Mitsui T, Endo I, Oshima Y, Kunishige M, Matsumoto T. Dermatomyositis with peripheral nervous system involvement: activation of vascular endothelial growth factor (VEGF) and VEGF receptor (VEGFR) in vasculitic lesions. Intern Med 2003;42:1233-9.

9. Wang Y, Cui LY, Chen L, Liu MS, Qi X, Li BH, et al. Nerve conduction studies in patients with dermatomyositis or polymyositis. Chin Med J (Engl) 2010;123:523-6.

10. Bohan A, Peter JB, Bowman RL, Pearson CM. Computer-assisted analysis of 153 patients with polymyositis and dermatomyositis. Medicine (Baltimore) 1977;56:255-86.

11. Vogelgesang SA, Gutierrez J, Klipple GL, Katona IM. Polyneuropathy in juvenile dermatomyositis. J Rheumatol 1995;22:1369-72.

12. Nomura $M$, Watanabe $T$, Mikami $H$, Ishikawa $H$, Yasui $K$, Yamazaki T, et al. Adult dermatomyositis with severe polyneuropathy: does neuromyositis exist? Neurol Sci 2010; 31:373-6.

13. Nguyen TP, Bangert C, Biliciler S, Athar P, Sheikh K. Dermatomyositis-associated sensory neuropathy: a unifying pathogenic hypothesis. J Clin Neuromuscul Dis 2014;16:7-11.

14. Irie $T$, Shigeto H, Koge J, Yamaguchi H, Murai H, Kira JI. Dermatomyositis complicated with asymmetric peripheral neuritis on exacerbation: a case report and literature review. Clin Exp Neuroimmunol 2016;7:373-80.

15. Cojocaru IM, Socoliuc G, Sapira V, Bastian A, Alexianu M, Moldovan M. Dermatomyositis and polyradiculoneuritis, a rare association. Rom J Intern Med 2011;49:217-21.

16. França MC Jr, Faria AV, Queiroz LS, Nucci A. Myositis with sensory neuronopathy. Muscle Nerve 2007;36:721-5. 